\title{
Robot-assisted right ureteral polypectomy: A case report
}

\author{
Brandon T. Karmo, DO; Kenneth Lim, DO; ${ }^{\dagger}$ Richard A. Santucci, MD; Sabry Mansour, MD ${ }^{\varsigma}$
}

*Lake Erie College of Osteopathic Medicine, Erie, PA; †Department of Urology, Detroit Medical Center, Detroit, MI; §Mclaren Lapeer Regional Medical Center, Lapeer, MI

Cite as: Can Urol Assoc J 2013;7(5-6):e426-9. http://dx.doi.org/10.5489/cuaj.1392 Published online June 12, 2013.

\section{Abstract}

Ureteral polyps are a rare cause of ureteral obstruction in the adult and pediatric populations. Fibroepitheial polyps (FEP) are the most common type of ureteral polyps. This clinical entity is very rare, warranting periodic clinical review by practitioners, and new advancements in laparoscopy allow new surgical approaches to its cure. We present the case of a 20-year-old male with rightsided flank pain. He was found to have right uretero-pelvic junction (UPJ) obstruction and subsequently underwent laparoscopic robotic-assisted right collecting system exploration, excision of polyps and right ureteropyeloplasty. Ureteral polyps were excised and determined to be fibroepithelial in origin based on the pathological report. Our case highlights the importance of having FEP in the differential diagnosis of ureteral obstruction. We also found that laparoscopic robot-assisted polypectomy is a safe and acceptable surgical option for the excision of ureteral polyps.

\section{Introduction}

Ureteral obstruction is a common challenge faced by many urologists. Nephrolithiasis, crossing vessels and ureteral strictures are the most common causes of ureteral obstruction. Ureteral polyps are an uncommon cause of urinary obstruction and only 236 cases of FEP have been reported since $1932 .{ }^{1}$ However, ureteral polyps can potentially grow large enough to cause obstructive symptoms and should be included in the differential diagnosis.

Fibroepithelial polyps (FEP) usually occur in adults between 20 and 40 years of age. ${ }^{2}$ They can arise anywhere between the renal pelvis and the urethra. Most occur in the renal pelvis near the uretero-pelvic junction (UPJ) and can lead to hematuria, colicky flank pain or dysuria. ${ }^{3}$ These symptoms are similar to other common causes of UPJ obstruction and can make the diagnosis of FEP difficult.

Histologically, most primary ureteral tumours are non- epithelial in origin and arise from mesoderm. FEP are benign and comprised of a richly vascular stroma with fibrous connective tissue. They are surrounded by normal transitional urothelium and it is for this reason that FEP are undetectable by urine cytology. Macroscopically, FEP are typically are 1 to $5-\mathrm{cm}$ in length and most often smooth, elongated and cylindric. ${ }^{4}$ They generally appear as multiple finger-like projections arising from a single base and have the ability to grow large enough to cause an obstruction. However, because of their slow growth, the resulting hydronephrosis is often less than expected. ${ }^{5}$

In the past, FEP treatment consisted of open surgical exploration and resection. However, as more non-invasive endoscopic techniques evolved, there are more successful reports of ureteroscopy to treat for ureteral polyps. ${ }^{6-8}$ Recently, the advent of robotic-assisted surgery has been successfully applied to treat benign ureteral disease. ${ }^{9}$ We discuss the clinical presentation, imaging, histological features, and the use of the daVinci surgical system to excise FEP.

\section{Case report}

A 20-year-old male with a history of recurrent renal calculi presented to the clinic with right flank pain. He denied fever, chills, weight loss, hematuria, frequency or dysuria. His medical history is significant for recurrent nephrolithiasis and most recently included a 4-mm calcium oxalate calculus in the proximal right ureter, as well as 2 lower pole right renal calculi that spontaneously passed in July 2011. His surgical history included extracorporeal shock wave lithotripsy (ESWL) on the right, cystoscopy, right ureteroscopy, right stone extraction, and right ureteral stent placement in December 2010.

Physical examination revealed right lower quadrant abdominal tenderness, but otherwise was within normal limits. Laboratory data revealed creatinine of 1.06 and all other biochemical data were within normal limits. Urinanalysis was unremarkable, with no evidence of infection or hematuria.

The patient underwent a computed tomography (CT) scan 
which revealed moderate hydronephrosis on the right side with concern for obstruction at the UPJ (Fig. 1). Intravenous pyelogram with nephrotomography showed persisting rightsided pelvicaliectasis with abrupt narrowing of the UPJ on the right, but no obstructing mass was seen (Fig. 2). The proximal right ureter distal to the UPJ was unremarkable. Nuclear renal scan showed evidence of apparent incomplete UPJ stenosis of the right collecting system (Fig. 3) and discrepant percent activity with $57 \%$ of the activity on the left and $43 \%$ on the right (Fig. 4). It is important to note that FEP were not apparent in any of our imaging modalities. Based on the results of our imaging and clinical exam, we speculated that the cause of our patient's UPJ obstruction was secondary to stricture formation.

Based on the imaging studies and clinical presentation, a diagnosis of right UPJ obstruction was made and the patient was scheduled for a laparoscopic robotic-assisted right collecting system exploration with right ureteropyeloplasty on April 3, 2012. The patient was placed in the right flank position and a Veress needle was placed into the peritoneal cavity and insufflation was accomplished up to $15 \mathrm{~cm}$ of water pressure. The trocars were placed in the peritoneal cavity in the modified lateral approach. Two 8-mm robotic trochars, a 12-mm camera port, and a 2-mm assistant port were used during the case. A 5-mm port was also inserted to place a grasper along the abdominal sidewall for slight superior retraction of the liver to expose the superior aspect of the kidney. Dissection of the pre-renal space was carried out inferiorly to identify the right ureter, which was traced proximally toward the UPJ and the renal pelvis was identified. The pelvis appeared to be dilated after exposure



Fig. 1. A computed tomography of the abdomen/pelvis without contrast showing right hydronephrosis. although the transition between the pelvis and the ureter did not reveal any evidence of obstruction to suggest UPJ stenosis. There was also no evidence of a crossing vessels causing UPJ junction. Due to the dilation of ureter on preoperative imaging, the decision was made to perform an Y-V plasty. Y-V plasty offers the advantage of maintaining continuity of the posterior wall of the ureter. During the opening of the renal pelvis, the proximal right ureter was exposed at which point several polyps were identified causing some intrinsic obstruction of the right proximal ureter. After spatulation of the ureter, we were able to identify 3 separate benign appearing polyps that were excised using sharp dissection. Robotic polypectomy did not change the closure of the renal pelvis. The renal pelvis was repaired using interrupted non-absorbable sutures. The robot had been docked in the standard fashion after placement of the trocars and the robotic instruments were used for the entire dissection and resection of these polyps.

An antegrade right ureteral stent was placed and the polyps were sent to the pathologist for examination. The pathology report revealed right fibroepithelial polyps. Microscopically, the polyps were covered by normal urothelium. The core of the polyp is composed of loose edematous stroma with a few lymphocytes. The patient was sent home on postoperative day 2 and the stent is scheduled to be removed within the following 3 to 6 months.

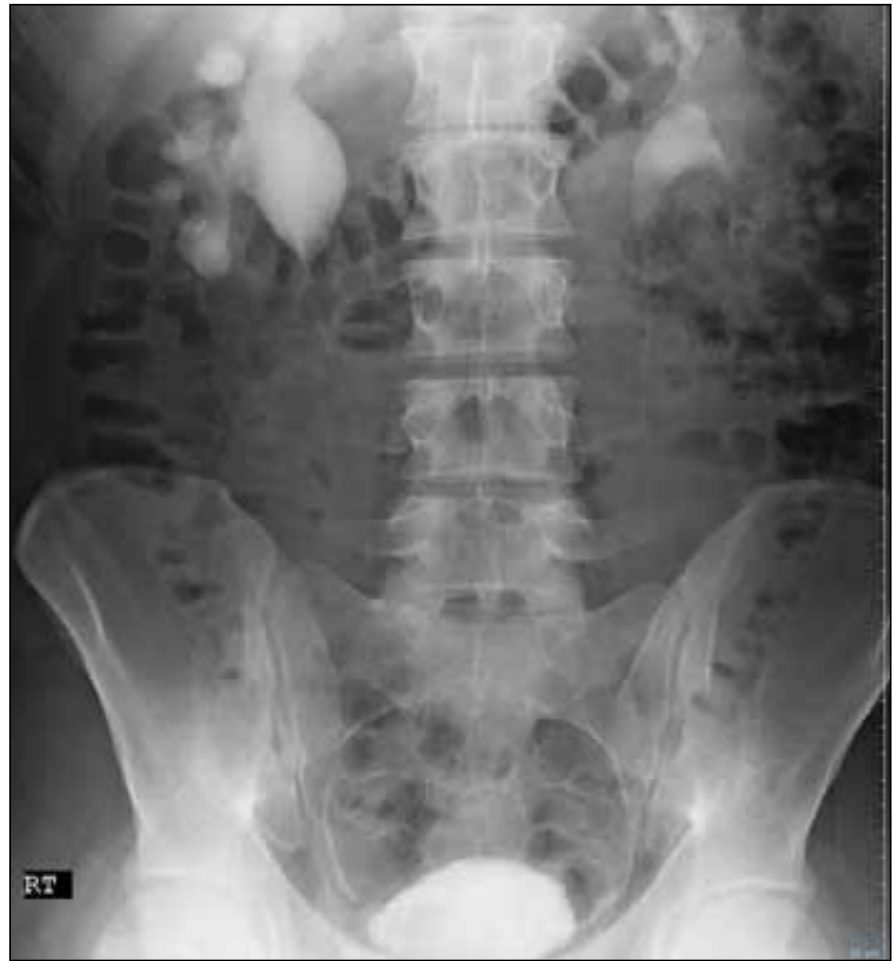

Fig. 2. An intravenous pyelography with nephrotomography showing right-sided pelvicaliectasis with abrupt narrowing of the uteropelvic junction on the right with no obstructing mass seen. 


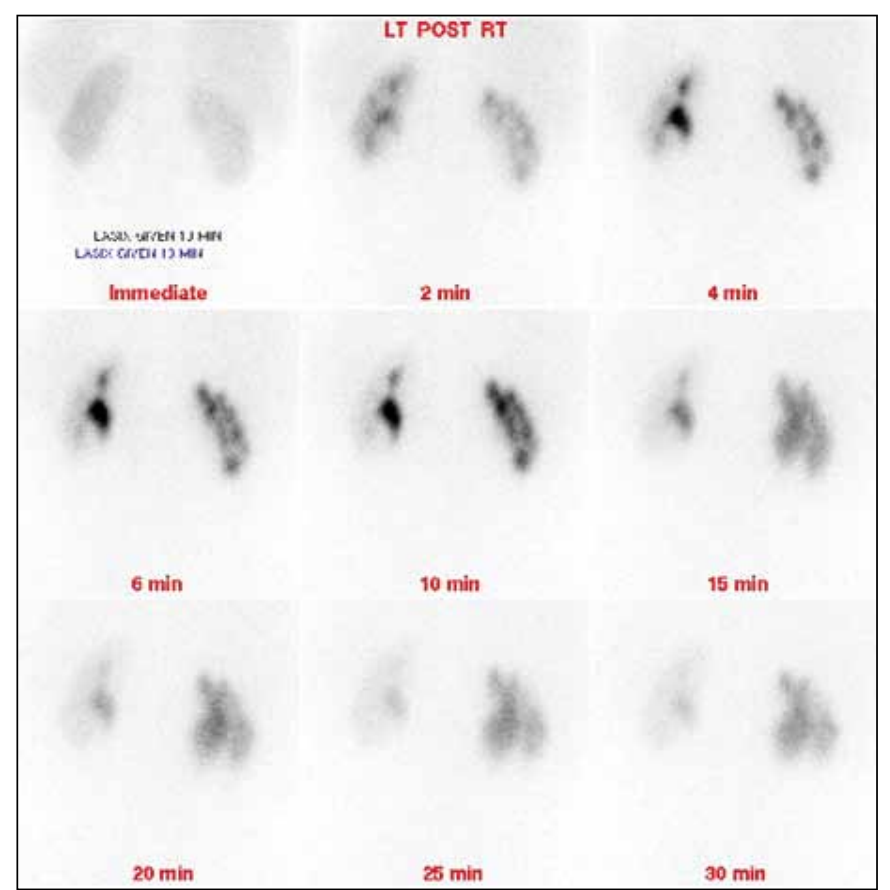

Fig. 3. A nuclear renal scan showing incomplete ureteropelvic junction stenosis of right collecting system.

\section{Discussion}

FEP present a unique challenge due to the similar symptoms they share with other more common causes of UPJ obstruction. Our case presentation highlights the importance of having FEP in the differential diagnosis of ureteral obstruction.

The etiology of FEP is believed to be likely secondary to developmental defects, infections, chronic irritation or trauma. Our initial differential diagnosis included stricture formation based on our patient's history of recurrent nephrolithiaisis, ESWL on the right, cystoscopy, right ureteroscopy, right stone extraction and right ureteral stent placement. After discovering the FEP intraoperatively, we determined that the cause of the UPJ obstruction was secondary to FEP.

Along with chronic irritation, FEP can also arise as congenital slow growing lesions. ${ }^{10}$ For this reason, it is possible that our patient's history of recurrent nephrolithiais may have been caused by FEP induced hydronephrosis. It is also possible that our patient's recurrent nephrolithiasis lead to chronic irritation which caused FEP to develop.

On intravenous pyelogram (IVU) or retrograde urograms (RU), FEP appear to be long, smooth ureteral filling defects and are also associated with varying degrees of hydronephrosis. ${ }^{10}$ It is crucial to distinguish FEP from other upper urinary tract carcinomas, such as transitional cell carcinoma, because the management and prognosis can be significantly different. Unfortunately, many FEP are missed on radiographical studies - this was the case with our patient who underwent both a CT of abdomen/pelvis and IVU. On IVU,

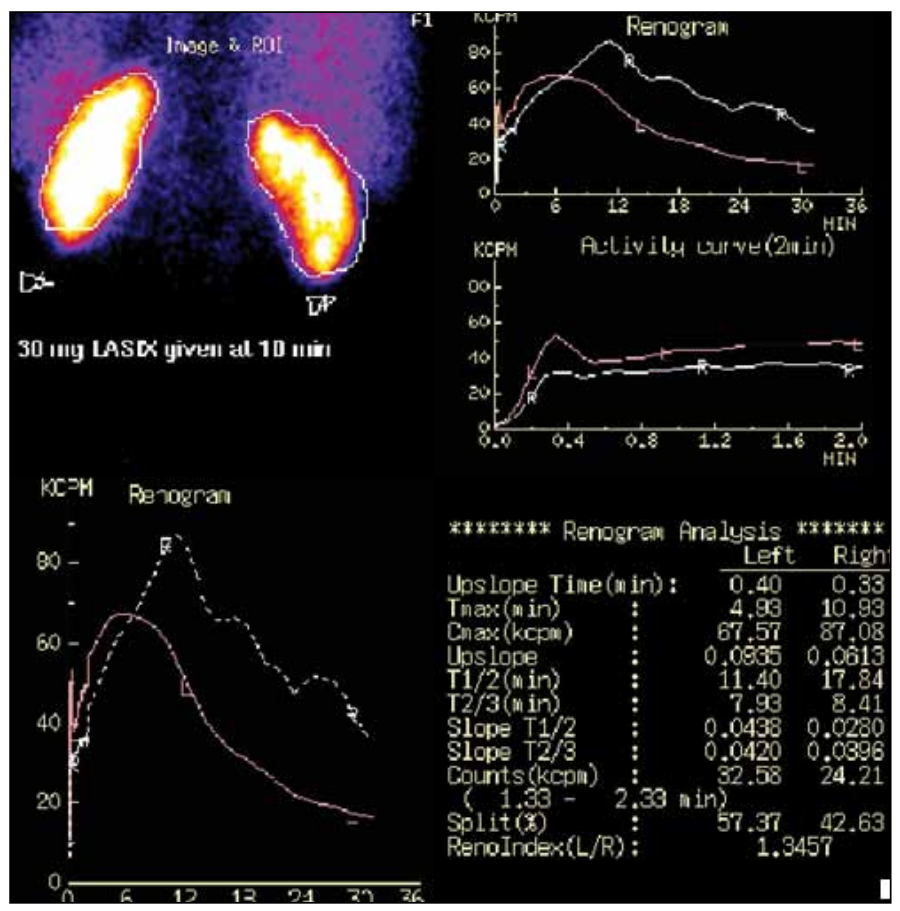

Fig. 4. A renogram reveals discrepant percent activity with $57 \%$ of the activity on the left and $49 \%$ on the right.

hydronephrosis is seen in $59 \%$ of cases. Filling defects are detected in $26 \%$ and $48 \%$ of cases by IVU and RU, respectively. ${ }^{11}$ The difficulty identifying ureteral polyps highlights the importance of including polyps in the differential diagnosis of ureteral obstruction.

Ureteral polyps have historically been managed by an open surgical approach and resection. With the advent of new technology over the years, many reports have described successful polypectomy through the use of ureteroscopy and endoscopic treatment. ${ }^{5}$ Holmium laser excision is another modality for endoscopic resection. We believe that ureteroscopy is a safe and effective modality for the removal of polyps. However, laparoscopic robot-assisted surgery offers the advantage of having increased working space. This allows for an easier excision of multiple ureteral polyps or those that may be too large to be removed by endoscopic surgery.

\section{Conclusion}

In our experience using the daVinci robotic system, we found that laparoscopic robot-assisted polypectomy is safe and effective for patients with refractory UPJ obstruction. The advantages of robotic-assisted surgery include clear intraoperative visualization and complete resection of the ureteral polyps. It also offers all of the benefits of minimally invasive surgery, such as small incisions, minimal blood loss, fast recovery and optimized cosmetic outcomes..$^{5}$ Therefore, laparoscopic robot-assisted polypectomy should be considered an acceptable surgical option to treat FEP. 
Competing interests: None declared.

This paper has been peer-reviewed.

\section{References}

1. Melicow MM, Findlay HV. Primary benign tumors of ureter: review of literature and report of case. Surg Gynecol Obstet 1932;54:680-9.

2. Mariscal A, Mate JL, Guasch I, et al. Cystic transformation of a fibroepithelial polyp of the renal pelvis: radiologic and pathologic findings. AJR Am J Roentgeno/1995;164:1445-6. http://dx.doi.org/10.2214/air.164.6.7754889

3. Williams TR, Wagner BJ, Corse WR, et al. Fibroepithelial polyps of the urinary tract. Abdom Imaging 2001;27:217-21. http://dx.doi.org/10.1007/s00261-001-0066-z

4. Harvin HJ. Ureteral Fibroepithelial Polyp on MDCT Urography. AJR Am J Roentgenol 2006;187:W434-5.

5. Bian Z, Liu X, Hua Y, et al. Laparoscopic Management of Multiple Ureteral Polyps in Children. J Urol 2011;186:1444-9. http://dx.doi.org/10.1016/i.juro.2011.05.056
6. Lam JS, Bingham JB, Gupta M. Endoscopic treatment of fibroepithelial polyps of the renal pelvis and ureter. Urology 2003;62:810. http://dx.doi.org/10.1016/S0090-4295(03)00691-5

7. Yagi S, Kawano Y, Gotanda T, et al. Endoscopic treatment of a long fibroepithelial ureteral polyp. Int $J$ Urol 2001;8:467. http://dx.doi.org/10.1046/j.1442-2042.2001.00343.x

8. Carey RI, Bird VG. Endoscopic management of 10 separate fibroepithelial polyps arising in a single ureter. Urology 2006;67:413. http://dx.doi.org/10.1016/j.urology.2005.08.020

9. Abaza R, Zafar S. Techniques for Laparoscopic and Robotic Localization of Intraluminal Ureteral Pathology. Urology 2008;180:266-70.

10. Turunc T, Kuzgunbay B, Canpolat T. Ureteral fibroepithelial polyps with calculi: a case series. J Med Case Rep 2008;2:280. http://dx.doi.org/10.1186/1752-1947-2-280

11. Tekdogan UY, Canakli F, Aslan Y, et al. Bilateral ureteral fibroepithelial polyps and review of the literature. Int J Urol 2005; 12: 98-100.

Correspondence: Dr. Richard Santucci, Harper Professional Building, Suite 1017, 4160 John R. Detroit, MI 48201; fax: 313-745-8222; rsantucc@DMC.org 